\title{
Origin of Local Structures of U-Co Melts: A First-Principles Study
}

\author{
Huanqing Zhang, Honggang Sun*, Qiutong Li and Li Wang * \\ School of Mechanical, Electrical and Information Engineering, Shandong University, Weihai, China
}

The local structures of U-Co melts have been studied by first-principle calculations. Two sub-peaks are observed in the first peaks of U-U pair distribution functions. The Voronoi polyhedral analyses also show two separate core-shell $U-U$ distances. Therefore, the calculated results propose that $U$ atoms will play dual roles, "chemical" and "topological", in the local structures of $U$-Co melts. In addition, the chemical effect of $U$ atoms will be strengthened when containing more $U$ atoms. The interaction of $\mathrm{Co}$ and $U$ atoms is slightly affected by the compositions. The Co-centered clusters are mostly prism-like or antiprismlike polyhedral, which can be predicted by the solute-solvent model. The distribution of the coordinated numbers of $\mathrm{Co}$ atoms is much narrower than that of $U$ atoms, showing relatively stable Co-centered clusters. The chemical and topological roles of $U$ atoms are intuitively observed in the electron density of U-Co melts, which presents both metallic and covalent bonding characteristics for $U-U$ bonds. In the end, we conclude that the partial localization of $U 5 f$-electron is responsible for the dual roles of $U$ atoms. The present results provide a theoretical understanding of the origin of the local structures of U-Co melts.

Edited by:

Ran $\mathrm{Li}$,

Beihang University, China

Reviewed by:

Jiang Ma,

Shenzhen University, China

Ruijuan Xiao,

Institute of Physics (CAS), China

*Correspondence:

Honggang Sun

sunhg@sdu.edu.cn

Li Wang

wanglihxf@sdu.edu.cn

Specialty section: This article was submitted to

Structural Materials,

a section of the journal

Frontiers in Materials

Received: 24 November 2021

Accepted: 23 December 2021

Published: 13 January 2022

Citation:

Zhang $H$, Sun $H, L i Q$ and Wang $L$ (2022) Origin of Local Structures of U-

Co Melts: A First-Principles Study.

Front. Mater. 8:821306.

doi: 10.3389/fmats.2021.821306
Keywords: U-Co melt, local structure, first-principle, SROs, bonding

\section{INTRODUCTION}

Uranium-based materials have been used in energy power, aerospace, biomedical instrument, and military weapons due to their high hardness and radioactivity. However, the corrosion resistance and process ability of the pure $\mathrm{U}$ metals are poor to limit their applications. Alloying is one of the effective methods to improve the properties of U metals. For example, U-Mo alloys firstly studied by Argonne National Laboratory present a good radiation resistance (Wiencek and Prokofiev, 2000; Hofman et al., 2003). Small amounts of Ti can improve the mechanical properties and the corrosion resistance of U-Ti alloys, which are considered as substitutes for W alloys (Zurek, 1994; Zurek, 1999; Shi et al., 2015; Li et al., 2020). U-Nb alloys with moderate Nb contents keep a high density compared to the pure U metals and show a good corrosion resistance (Manner et al., 1999; Orlov, 2002). Furthermore, Huang et. al. exploited U-Co-Al and U-Co-B metallic glasses (MGs) by melt-spin quenching method, in which the corrosion resistance is better than that in crystal alloys (Huang et al., 2016; Xu et al., 2018). The better performance can be contributed to homogeneity and no grain boundaries of the amorphous alloys.

However, the glass-forming ability (GFA) of U-based alloys is poor and the design of alloy compositions is still confusing. $\mathrm{U}-\mathrm{M}(\mathrm{M}=\mathrm{Co}, \mathrm{Fe}, \mathrm{Ni}, \mathrm{Cr}) \mathrm{MGs}$ were prepared by Hammer-and-Anvil technique (HA) (Giessen and Elliott, 1978; Elliott et al., 1980; Elliott et al., 1981; Elliott and Giessen, 1982) and melt-spinning technique (MS) (Drehman, 1985; Drehman and Poon, 1985; Poon et al., 1985), which are mostly amorphous ribbons rather than bulk MGs. Elliott et. al. prepared U-Fe MGs by liquid quenching and irradiation techniques, but there were some crystal phases in materials (Elliott et al., 1980). Previously, Dreham's work noted that the U-Co alloy's composition with the best 
GAF was $\mathrm{U}_{72} \mathrm{Co}_{48}$ based on the differential scanning calorimeter analysis (Drehman and Poon, 1985; Poon et al., 1985). Huang et. al. noted that the amorphous phase was most readily formed in the vicinity of $\mathrm{U}_{66.7} \mathrm{Co}_{33.3}$ composition (Huang et al., 2020). It is well known that the GFA of alloys is related to the structural properties of melts because most MGs are prepared by supercooling melts. Therefore, the understanding of local structures of U-based melts is in favor of designing alloy composition to get better U-based MGs. Theoretical researches have been used to analyze the local structures of U-based alloy or MGs. Xiang et. al. calculated the occupied positions of $\mathrm{Nb}$ in $\gamma$-U crystal, which related the structural stability to the charges distribution (Xiang et al., 2008). Recently, Zhang et. al. have studied the atomic structure of U-Fe MGs based on reverse Monte Carlo simulations (Zhang et al., 2012). The work revealed the topological and chemical local structures of $\mathrm{U}$-centered and Fe-centered clusters, and noted the structural complexity of these MGs. However, the relevant studies of local structures of $\mathrm{U}$-based melts are still scarce, and there is an urgent need for theoretical work.

In the present work, the local structures of U-Co melts $\left(\mathrm{U}_{50} \mathrm{Co}_{50}, \mathrm{U}_{67} \mathrm{Co}_{33}\right.$, and $\left.\mathrm{U}_{86} \mathrm{Co}_{14}\right)$ are studied by first-principle dynamics calculations. Several structural indicators, such as pair distribution function, Voronoi polyhedra, and coordination number, are used to correlate the structural characteristics with melt compositions. At last, we try to reveal the origin of the special structures in the U-Co melts by studying the atomic bonding characteristic. The present work will provide a theoretical view to understand the local structures of U-Co melts.

\section{MATERIALS AND METHODS}

The first-principle dynamics calculations were performed by Vienna Ab-initio Simulation Package (VASP) (Kresse and Furthmüller, 1996). The projector augmented wave method (PAW) was used to treat the core electrons (Blöchl, 1994; Kresse and Joubert, 1999). The valence electronic configurations of $\mathrm{U}$ and $\mathrm{Co}$ atoms were $5 f^{3} 6 s^{2} 6 p^{6} 6 d^{1} 7 s^{2}$ and $3 s^{8} 4 p^{1}$, respectively. The exchange and correlation interactions were dealt with the GGA-PBE functional (Perdew et al., 1996). The plane wave cut-off energy was $350 \mathrm{eV}$. Cubic supercells containing 200 atoms with periodic boundary conditions were constructed by a random distribution of $U$ and Co atoms, which were used to act as the original structures for the subsequent dynamic process. The densities of the cubic supercells are determined by Drehman's experiment (Drehman and Poon, $1985)$, which are $14.66,16.27$, and $17.49 \mathrm{~g} / \mathrm{cm}^{3}$ for $\mathrm{U}_{50} \mathrm{Co}_{50}$, $\mathrm{U}_{67} \mathrm{Co}_{33}$, and $\mathrm{U}_{86} \mathrm{Co}_{14}$. Then the densities of the different $\mathrm{U}$-Co systems were adjusted by the zero external pressure method. Only the $\Gamma$ point was used to sample the supercell Brillouin zone. The canonical ensemble (NVT) was used to perform the dynamic calculations. The temperature was controlled through a Nosé thermostat (Nosé, 1984). To achieve liquid U-Co systems, the supercells were firstly well equilibrated for 45 ps with 3 fs time step at $2000 \mathrm{~K}$. Then the temperature of the systems was reduced to $1,500,1,200$, and

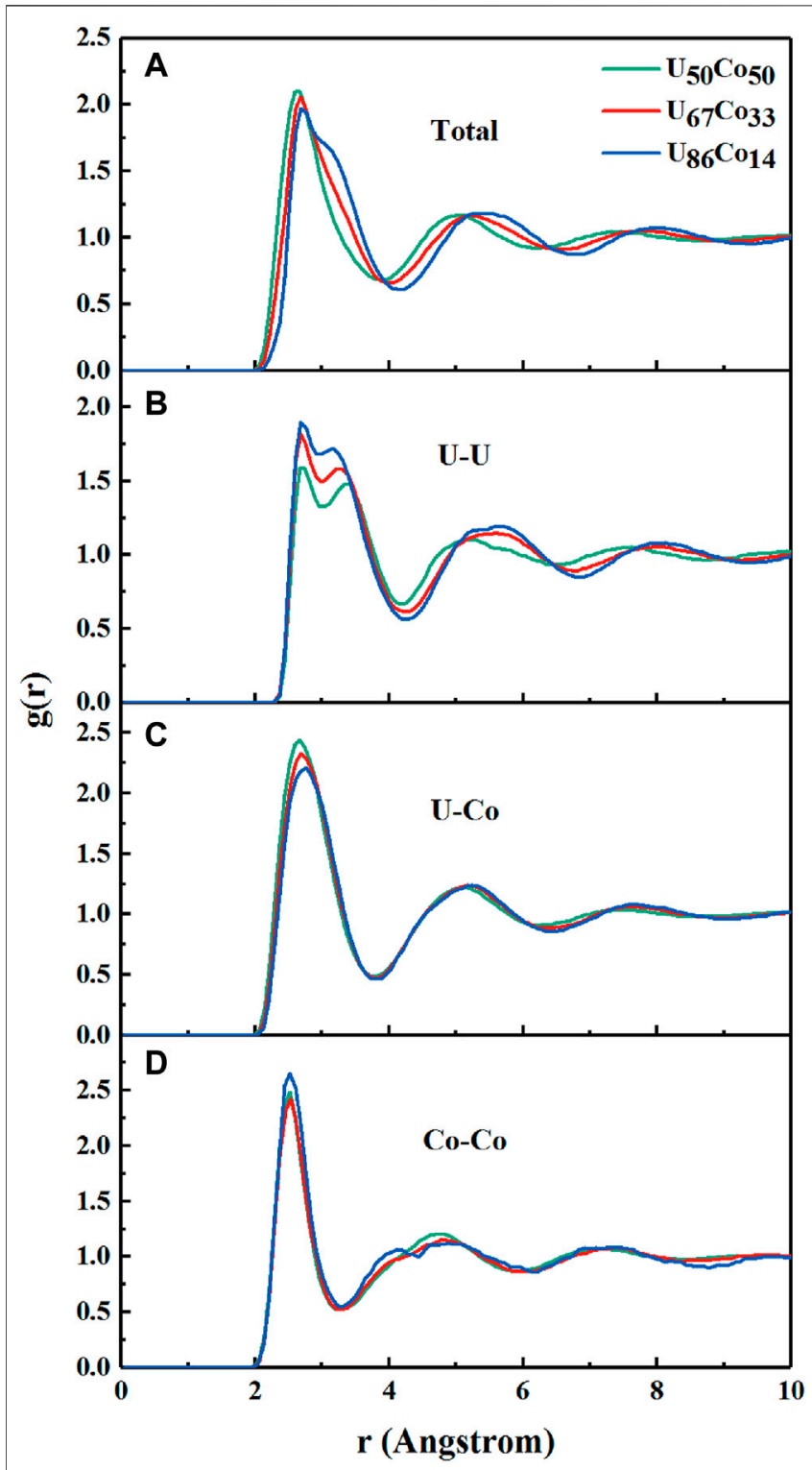

FIGURE 1 | The pair distribution functions of (A) Total, (B) U-U, (C) $\mathrm{U}-\mathrm{Co}$, and (D) Co-Co for $\mathrm{U}_{50} \mathrm{Co}_{50}, \mathrm{U}_{67} \mathrm{CO}_{33}$, and $\mathrm{U}_{86} \mathrm{CO}_{14}$ melts.

$1500 \mathrm{~K}$ for $\mathrm{U}_{50} \mathrm{Co}_{50}, \mathrm{U}_{67} \mathrm{Co}_{33}$, and $\mathrm{U}_{86} \mathrm{Co}_{14}$, which are about $200 \mathrm{~K}$ higher than the melting points based on the U-Co phase diagram (Massalski et al., 1990). At the temperature, the structures were also equilibrated for 45 ps with the time step of $3 \mathrm{fs}$, and the last 5,000 dynamic configurations were employed to analyze the structural characteristic.

\section{RESULTS}

\section{Pair Distribution Function}

The structures of U-Co melts are described by the total and partial pair distribution function (PDF). As shown in Figure 1, the PDFs, $\mathrm{g}_{\text {Total }}(\mathrm{r}), \mathrm{g}_{\mathrm{U}-\mathrm{U}}(\mathrm{r}), \mathrm{g}_{\mathrm{U}-\mathrm{Co}}(\mathrm{r})$, and $\mathrm{g}_{\mathrm{Co}-\mathrm{Co}}(\mathrm{r})$, show a liquid structural 


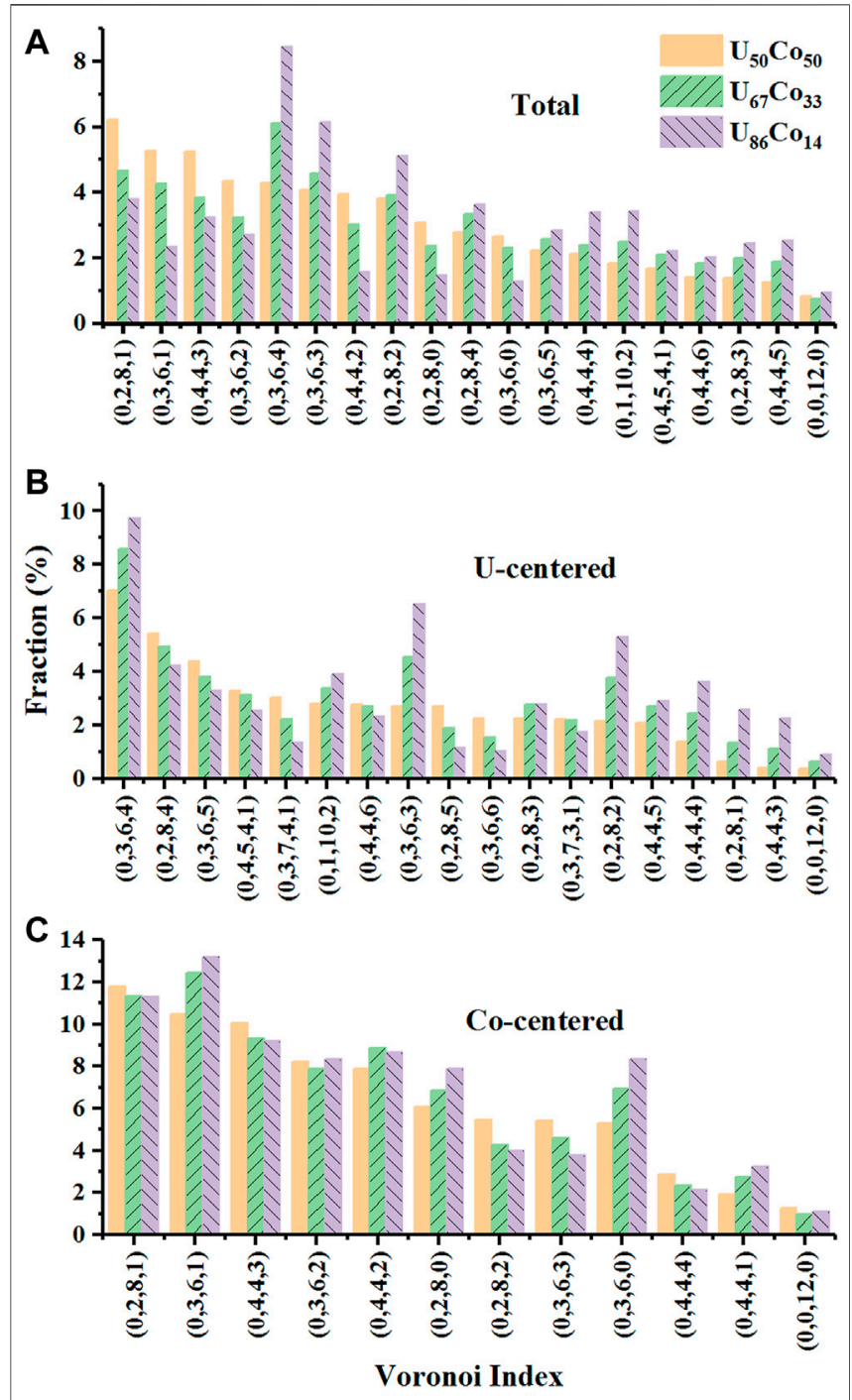

FIGURE 2 | The distributions of (A) total, (B) U-centered, and (C) Cocentered Voronoi polyhedron for $\mathrm{U}_{50} \mathrm{Co}_{50}, \mathrm{U}_{67} \mathrm{Co}_{33}$, and $\mathrm{U}_{86} \mathrm{Co}_{14}$.

characteristic. Generally, the first peaks of PDFs correspond to the short-range orders (SROs). It can be seen from Figure 1 $\mathbf{A}$ that the positions of the first peaks of the total PDFs are at about 2.63, 2.66, and $2.73 \AA$ for $\mathrm{U}_{50} \mathrm{Co}_{50}, \mathrm{U}_{67} \mathrm{Co}_{33}$, and $\mathrm{U}_{86} \mathrm{Co}_{14}$, respectively. The right-shifts of the peaks are also observed for the second peaks at about 4.92, 5.07, and $5.19 \AA$ for $\mathrm{U}_{50} \mathrm{Co}_{50}, \mathrm{U}_{67} \mathrm{Co}_{33}$, and $\mathrm{U}_{86} \mathrm{Co}_{14}$ melts, respectively. This is in agreement with the larger atomic radii of about $1.56 \AA$ of $\mathrm{U}$ atoms than $1.25 \AA$ of Co atoms (Porterfield, 1984; Kaye and Laby, 1993), which may present larger and more open short-range configurations. Importantly, with increasing $U$ concentrations, the heights of the first peaks become smaller, while the shoulder peaks at about 3.1-3.2 $\AA$ are more significant. The phenomenon implies the complexity of the SROs of U-Co melts.

As shown in Figure 1B, the first peaks of the three U-U PDFs are splitting into two sub-peaks. Hereinto, the positions of the first sub-peaks (U-U) ${ }_{1}$ are hardly affected by the compositions, which are all localized at about $2.74 \AA$. The value is much smaller than $3.12 \AA$ used in the hard spheres model, while it is close to the $\mathrm{U}$-U bond length of about $2.77 \AA$ measured by Bowen (Bowen and Sutton, 1958). The second sub-peaks (U-U) $)_{2}$ are at about $3.21,3.13$, and $3.08 \AA$ for $\mathrm{U}_{50} \mathrm{Co}_{50}, \mathrm{U}_{67} \mathrm{Co}_{33}$, and $\mathrm{U}_{86} \mathrm{Co}_{14}$ melts, respectively. These positions are close to the results of the hard spheres model and are in agreement with the shoulder peaks of the total PDFs. Therefore, we conclude that the splitting of the first peaks originates from the double-shell U-centered clusters with separate $U-U$ bond lengths. The splitting peaks are also observed in the liquid uranium (del Rio et al., 2020) and the CeCe peaks of the high-density Ce-Al alloy (Sheng et al., 2007), and are similar to the asymmetric shape of the peak in $\mathrm{Cu}-\mathrm{Zr}$ alloy (Ding et al., 2014). It should be emphasized that the (U-U) distance of $2.74 \AA$ is even shorter than the U-U bond lengths (2.8-3.2 $\AA$ ) in U/Co metals and intermetallic compounds (Baenziger et al., 1950). Namely, some U atoms show a strong chemical effect. Therefore, in U-Co melts, the $\mathrm{U}$ atoms play two roles: one is the "chemical sphere" while another is the "topological sphere", which will markedly influence the SROs of U-Co melts. The chemical effect is also observed between $\mathrm{U}$ and Co atoms. As shown in Figure 1C, the positions of the first peaks of U-Co PDFs are at about 2.64-2.76 $\AA$, which are smaller than $2.81 \AA$ based on the hard spheres model and 2.78-2.90 $\AA$ in U/Co intermetallic compounds (Baenziger et al., 1950). Therefore, the effect of Co atoms in the U-Co melts is similar to the Fe atoms in U-Fe alloy, which play a certain chemical role (Zhang et al., 2012). However, the positions of the first peaks of Co-Co PDFs are fixed at about $2.50 \AA$ as shown in Figure 1D, which are equal to the result of the hard spheres model. The results suggest that both the chemical and the topological factors play an important role in the SROs of U-Co melts.

\section{Voronoi Polyhedra}

The Voronoi polyhedral analyses are employed to further illuminate the topological characteritics of the SROs in different melts. As shown in Figure 2, only the polyhedra with more than $2 \%$ distributions are listed with different Voronoi indexs. Figure 2A gives the total distributions, where the top five polyhedra are $\langle 0,2,8,1\rangle,\langle 0,3,6,1\rangle,\langle 0,4,4,3\rangle$, $<0,3,6,2\rangle$, and $\langle 0,3,6,4\rangle$ for $\mathrm{U}_{50} \mathrm{Co}_{50},\langle 0,3,6,4\rangle,\langle 0,2,8,1\rangle$, $<0,3,6,3\rangle,\langle 0,3,6,1\rangle$, and $<0,2,8,2\rangle$ for $\mathrm{U}_{67} \mathrm{Co}_{33}$, and $\langle 0,3,6,4\rangle$, $<0,3,6,3\rangle,\langle 0,2,8,2\rangle,\langle 0,2,8,1\rangle$, and $\langle 0,2,8,4\rangle$ for $\mathrm{U}_{86} \mathrm{Co}_{14}$, respectively. With increasing $\mathrm{U}$ atoms, the quantities of large clusters such as $\langle 0,2,8,2\rangle,\langle 0,4,4,4\rangle,\langle 0,3,6,3\rangle,\langle 0,3,6,4\rangle$, $<0,1,10,2\rangle,\langle 0,2,8,4\rangle$ increase, while the quantities of small clusters such as $\langle 0,3,6,1\rangle,\langle 0,4,4,2\rangle,\langle 0,2,8,0\rangle,\langle 0,2,8,1\rangle$, $<0,4,4,3\rangle,\langle 0,3,6,2\rangle$ decrease. The result is agreement with the larger $\mathrm{U}$ atomic radius. As shown in Figures 2B,C, for all melts, the ideal "icosahedral ordering" $<0,0,12,0\rangle$ in both U- and Cocentered polyhedra are much less than $1 \%$, which influence to the SROs is slight. In the U-centered polyhedra, the icosahedra-like clusters such as Voronoi indexs $\langle 0,3,6,4\rangle,\langle 0,1,10,2\rangle,\langle 0,3,6,3\rangle$, $\langle 0,2,8,3\rangle, \quad\langle 0,2,8,2\rangle, \quad\langle 0,4,4,5\rangle, \quad\langle 0,4,4,4\rangle, \quad\langle 0,2,8,1\rangle$, and $<0,4,4,3>$ observably increase for the higher $U$ concentrations. Conversely, the Frank-Kasper polyhedra such as $\langle 0,2,8,4\rangle$, $\langle 0,3,6,5\rangle,\langle 0,4,5,4,1\rangle,\langle 0,3,7,4,1\rangle,\langle 0,2,8,5\rangle$, and $<0,3,6,6\rangle$ 
TABLE 1 | The positions of the first peaks of PDFs $(\AA)$, the positions of the two subpeaks in U-U PDFs $(\AA)$, and the calculated atomic radii $(\AA)$ are listed for $\mathrm{U}_{50} \mathrm{Co}_{50}, \mathrm{U}_{67} \mathrm{Co}_{33}$, and $\mathrm{U}_{86} \mathrm{Co}_{14}$ melts.

\begin{tabular}{lcccccccc}
\hline U-Co & Total & Co-Co & U-Co & $(\mathbf{U}-\mathbf{U})_{\mathbf{1}}$ & $(\mathbf{U}-\mathbf{U})_{\mathbf{2}}$ & $\boldsymbol{R}_{\mathbf{C o}}$ & $\boldsymbol{R}_{\mathbf{U}}$ & $\mathbf{R}_{\mathbf{C o} / \mathbf{U}}^{\star}$ \\
\hline $\mathrm{U}_{50} \mathrm{Co}_{50}$ & 2.63 & 2.50 & 2.64 & 2.74 & 3.21 & 1.25 & 1.39 & 0.90 \\
$\mathrm{U}_{67} \mathrm{Co}_{33}$ & 2.66 & 2.50 & 2.68 & 2.74 & 3.13 & 1.25 & 1.43 & 0.87 \\
$\mathrm{U}_{86} \mathrm{Co}_{14}$ & 2.73 & 2.50 & 2.76 & 2.74 & 3.08 & 1.25 & 1.51 & 0.83
\end{tabular}

decrease for the higher $U$ concentrations. The Co-centered $<0,2,8,1>$ polyhedra are canonical Kasper polyhedra, which also can be classified as defected icosahedra-like clusters. The polyhedra with indexs $\langle 0,3,6,2\rangle$ and $<0,4,4,3\rangle$ are based on the $<0,2,8,1>$ polyhedra with increasing defects in polytetrahedral packing. Interestingly, as shown in Figure 2C, the prism-like or antiprism-like packing polyhedra such as $\langle 0,3,6,0\rangle$ (tri-capped trigonal prism, TTP), $\langle 0,2,8,0\rangle$ (bi-capped square archimedean antiprism, BSAP), $<0,4,4,1>$, and $<0,3,6,1\rangle$ (distorted TTP and BSAP) gradually increase and become dominant from $\mathrm{U}_{50} \mathrm{Co}_{50}$, $\mathrm{U}_{67} \mathrm{Co}_{33}$, to $\mathrm{U}_{86} \mathrm{Co}_{14}$ melts, while the numbers of icosahedra clusters decrease.

In the previous Miracle's work (Miracle, 2004; Miracle, 2006), the radius ratios $\mathrm{R}$ (the solute atom radius normalized by the solvent atom radius) of the U-Co alloy should be 0.81 (atomic radius 0.158 for $\mathrm{U}$ and 0.128 for $\mathrm{Co}$ ). Therefore, the predicted Co concentration for the better glass-forming ability of U-Co binary alloy is less than $20 \%$ according to the Ma and Xu's idealized atomic packing model (Shi et al., 2008). However, the work by Egami and Waseda (Egami and Waseda, 1984) noted that the U-Co alloy might contain at least $21.8 \%$ Co to form metallic glasses by liquid quenching method. As mentioned above, the Cocentered polyhedra are mainly dense packing local structures, which types are slightly influenced by compositions. However, the U-centered polyhedra are mainly larger open Frank-Kasper polyhedra due to the larger $U$ atomic radius. This is the reason for keeping high Co concentrations in the experiments. Therefore, the decreasing of Co atoms will be disadvantage for the glassforming ability of U-Co alloys. On the other hand, the icosahedra-like U-centered polyhedra observably increase for the higher $U$ concentrations due to the enhanced chemical role of $U$ atoms. Namely, there is an equilibrium point of the quantity of the dense packing local structures when the $U$ concentration is increasing. Thus, the present work concludes the $\mathrm{U}_{67} \mathrm{Co}_{33}$ alloy may possess a better glass-forming ability, which is agreement with Huang's experiments (Huang et al., 2015; Huang et al., 2016; Xu et al., 2018; Xu et al., 2018).

Sheng et. al. (Sheng et al., 2006) presented the dependence of the local packing on atomic size ratio $\left(R^{\star}\right)$ based on the solutesolvent model (Miracle, 2004; Miracle, 2006). The method was used to analyze the dependence of the Co- centered clusters on atomic size ratio $R^{\star}$. Sheng's calculations gave the icosahedral type polyhedron with $R^{\star}$ around $1.2-0.902$, the BSAP type polyhedron with $R^{\star}$ around $0.902-0.835$, and the TTP type polyhedron with $R^{\star}$ around $0.835-0.732$. The actual sizes of atoms in a material depend upon the local chemistry and structure. In the present work, the chemical effect is

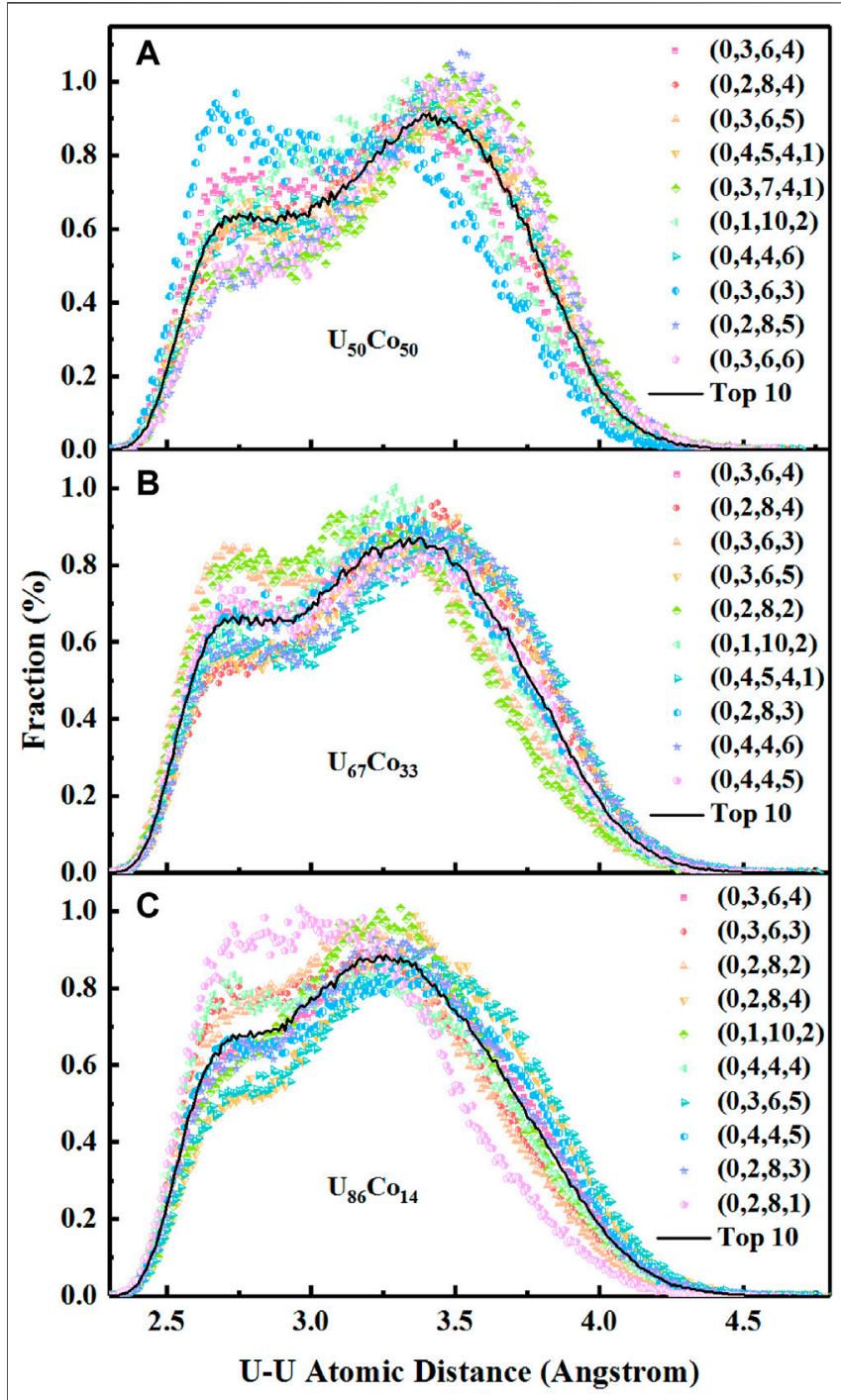

FIGURE 3 | The $U-U$ distances in the top ten Voronoi polyhedra of (A) $\cup_{50} \mathrm{Co}_{50}$, (B) $\cup_{67} \mathrm{Co}_{33}$, and (C) $\cup_{86} \mathrm{Co}_{14}$ melts.

introduced to correct the atomic sizes. For Co-centered clusters (Co solute and $U$ solvent), a half of the position of the first peak in $\mathrm{g}_{\mathrm{Co}-\mathrm{Co}}(\mathrm{r})$ is considered as the Co atomic size $\left(R_{\mathrm{Co}}\right)$, while the $\mathrm{U}$ atomic size $\left(R_{\mathrm{U}}\right)$ is calculated by subtracting $R_{\mathrm{Co}}$ from the position of the first peak in $\mathrm{g}_{\mathrm{U}-\mathrm{Co}}(\mathrm{r})$. Table 1 lists the calculated atomic size ratio $R_{\mathrm{Co} / \mathrm{U}}^{*}$ Combined with the above Voronoi polyhedral analyses of Co-centered clusters, the present calculations are in agreement with Sheng's results. When the constituents are closer to the solute-solvent model, namely more $U$ atoms and fewer Co atoms, the TTP type polyhedra become more dominant. The TTP-type polyhedra are usually observed in transition-metal-metalloid systems (Watson and Bennett, 1991), and the prism-like SROs are close to the coordination structure in $\mathrm{U}_{6} \mathrm{Co}$ intermetallic compound. Therefore, the role of the Co atoms in U-Co alloys is similar to the metalloid (such as $\mathrm{B}, \mathrm{C}$ and $\mathrm{Si}$ in $\mathrm{FeBC}, \mathrm{FeSiC}$ amorphous alloys (Ganesh and Widom, 2008; Pan et al., 2009; 
TABLE 2 | The NN coordination numbers (CNs), and the componential proportions around $\mathrm{U}$ and $\mathrm{Co}$ atoms ( $\mathrm{U}-\mathrm{CPs}$ and $\mathrm{Co}-\mathrm{CPs}$ ) in $\mathrm{U}-\mathrm{Co}$ melts are listed for $\mathrm{U}_{50} \mathrm{Co}_{50}, \mathrm{U}_{67} \mathrm{CO}_{33}$, and $\mathrm{U}_{86} \mathrm{Co}_{14}$.

\begin{tabular}{|c|c|c|c|c|c|c|c|}
\hline \multirow[t]{2}{*}{ U-Co } & \multicolumn{3}{|c|}{ CNs } & \multicolumn{2}{|c|}{ U-CPs } & \multicolumn{2}{|c|}{ Co-CPs } \\
\hline & Total & $\mathbf{U}$ & Co & $\mathbf{U}$ & Co & $\mathbf{U}$ & Co \\
\hline $\mathrm{U}_{50} \mathrm{Co}_{50}$ & 12.4 & 14.1 & 10.8 & $52.2 \%$ & $47.8 \%$ & $63.3 \%$ & $36.7 \%$ \\
\hline $\mathrm{U}_{67} \mathrm{CO}_{33}$ & 12.6 & 13.5 & 10.6 & $71.1 \%$ & $28.9 \%$ & $77.1 \%$ & $22.9 \%$ \\
\hline $\mathrm{U}_{86} \mathrm{Co}_{14}$ & 12.7 & 13.0 & 10.5 & $88.6 \%$ & $11.4 \%$ & $90.4 \%$ & $9.6 \%$ \\
\hline
\end{tabular}

Pan et al., 2015)). Namely, the Co atoms are the core of the chemical short range orders, which local structures will construct the bones of the U-Co melts.

We also try to trace the origin of the SROs of the U-centered clusters. Figure 3 gives the core-shell $U-U$ distances in the top ten Voronoi polyhedra. It can be seen that two separate peaks are present at about 2.70 and $3.34 \AA$ for $\mathrm{U}_{50} \mathrm{Co}_{50}, 2.70$ and $3.28 \AA$ for $\mathrm{U}_{67} \mathrm{Co}_{33}$, and 2.70 and $3.24 \AA$ for $\mathrm{U}_{86} \mathrm{Co}_{14}$, respectively, which are in keeping with the PDFs' results. For the icosahedral type polyhedron, there are more peaks with shorter $\mathrm{U}-\mathrm{U}$ distances (2.50-3.00 ̊). For the Frank-Kasper polyhedron, the peaks with shorter U-U distances are less. For example, the ratio of the shorter U-U distances is $33.3 \%$ in the icosahedral type polyhedron $<0,3,6,3\rangle$, while the ratio of the shorter $U-U$ distances is only $14.3 \%$ in the Frank-Kasper polyhedron $<0,2,8,4>$. In summary, the polyhedral analyses again indicate that two effects of $\mathrm{U}$ atoms in $\mathrm{U}$-Co melts are responsible for the special atomic local packing.

\section{Coordination Number}

The NN coordination numbers (CNs) and the componential proportions of $\mathrm{U}$ and $\mathrm{Co}$ atoms in partial $\mathrm{CNs}$ can be achieved from the PDFs using the first hollow as cutoff values for $\mathrm{U}_{50} \mathrm{Co}_{50}$, $\mathrm{U}_{67} \mathrm{Co}_{33}$, and $\mathrm{U}_{86} \mathrm{Co}_{14}$, respectively, which are listed in Table 2. The calculated $\mathrm{CNs}$ of $\mathrm{U}$ atoms are $14.1(7.3 \mathrm{U}+6.8 \mathrm{Co}), 13.5$ $(9.6 \mathrm{U}+3.9 \mathrm{Co})$, and $13.0(11.6 \mathrm{U}+1.4 \mathrm{Co})$ for $\mathrm{U}_{50} \mathrm{Co}_{50}, \mathrm{U}_{67} \mathrm{Co}_{33}$, and $\mathrm{U}_{86} \mathrm{Co}_{14}$, respectively. The calculated $\mathrm{CNs}$ of $\mathrm{Co}$ atoms are $10.8(6.8 \mathrm{U}+4.0 \mathrm{Co}), 10.6(8.2 \mathrm{U}+2.4 \mathrm{Co})$, and $10.5(9.5 \mathrm{U}+1.0$ Co) for $\mathrm{U}_{50} \mathrm{Co}_{50}, \mathrm{U}_{67} \mathrm{Co}_{33}$, and $\mathrm{U}_{86} \mathrm{Co}_{14}$, respectively. The larger partial $\mathrm{CNs}$ of $\mathrm{U}$ atoms agree with the Voronoi polyhedral analyses, which gives larger U-centered clusters (Frank-Kasper type) than the Co-centered clusters (BSAP and TTP type). Around $\mathrm{U}$ and $\mathrm{Co}$ atoms, the componential proportions of $\mathrm{U}$ atoms are 52.2 and $63.3 \%\left(\mathrm{U}_{50} \mathrm{Co}_{50}\right), 71.1$ and $77.1 \%\left(\mathrm{U}_{67} \mathrm{Co}_{33}\right)$, and 88.6 and $90.4 \%\left(\mathrm{U}_{86} \mathrm{Co}_{14}\right)$, which are all larger than the nominal proportions. The componential segregation can be contributed to the chemical effect between $\mathrm{U}$ and U/Co atoms. Moreover, more $\mathrm{U}$ atoms are around the Co atom than those around the $\mathrm{U}$ atom, suggesting that stronger $\mathrm{U}$-Co bonding than $\mathrm{U}-\mathrm{U}$ bonding. For higher $\mathrm{U}$ contents, the difference of $\mathrm{U}-\mathrm{U}$ and $\mathrm{U}$-Co bonding is weakened as a result of the participation of more $\mathrm{U}$ atoms in the chemical bonding.

Instead of the CNs results from PDFs, the CNs can be calculated by the statistics of the different Voronoi polyhedra. As shown in Figure 4, the method will give the distribution of the integral CNs. Unlike the crystal, each atom in the liquid/melt has

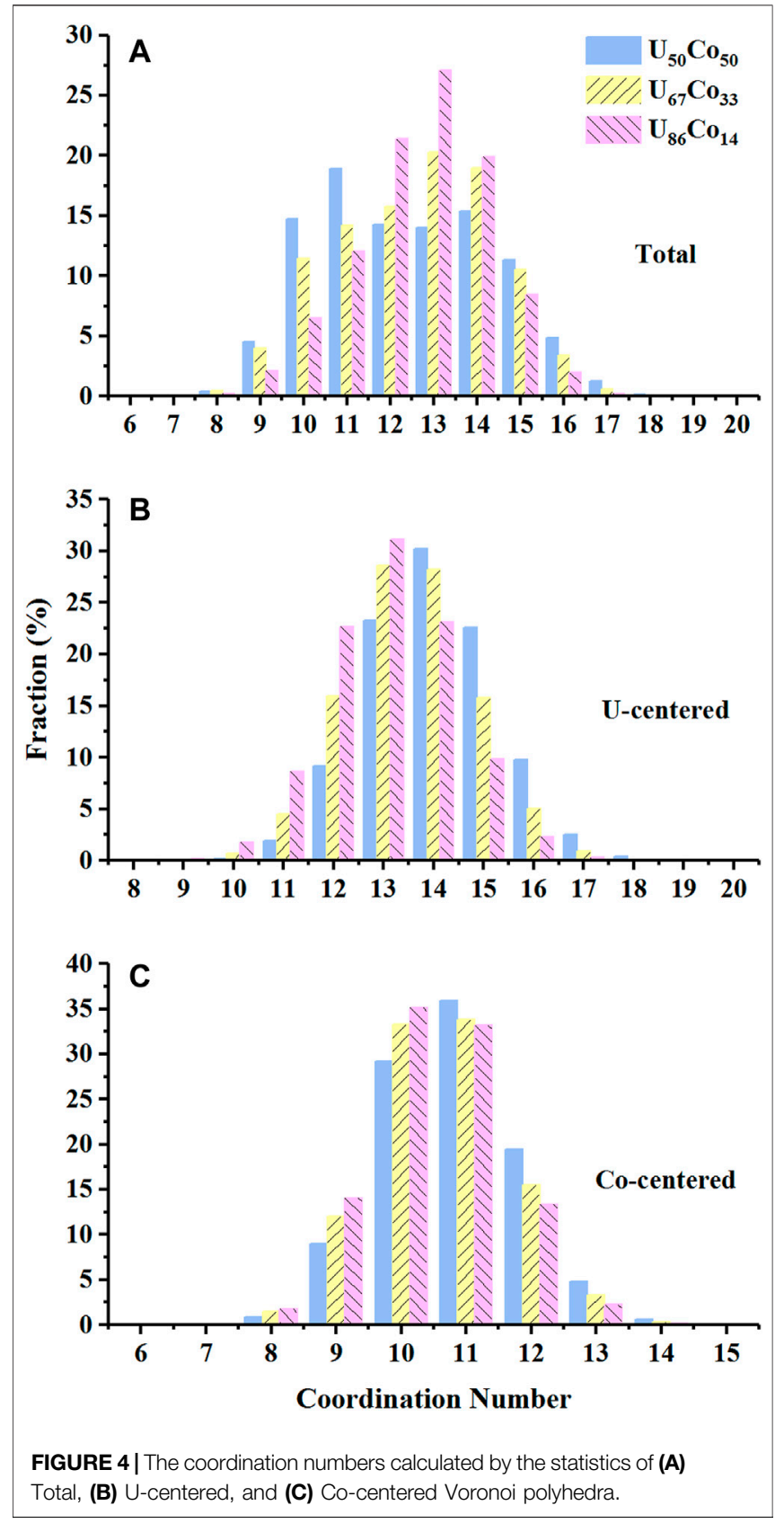

its own $\mathrm{CN}$. Therefore, the most probable $\mathrm{CN}$ represents the average structure and the favored SRO, whereas higher or lower CNs represent inevitable packing fluctuations (Egami, 2011). For $\mathrm{U}_{50} \mathrm{Co}_{50}$, the U-centered CNs mainly focus on 14 , while the Cocentered CNs mostly center on 11 . However, for $\mathrm{U}_{67} \mathrm{Co}_{33}$ and $\mathrm{U}_{86} \mathrm{Co}_{14}$, the proportions of the high CNs ( $\geq 14$ for U-centered, and $\geq 11$ for Co-centered) decrease while the proportions of the low CNs ( $\leq 13$ for $\mathrm{U}$-centered, and $\leq 10$ for Co-centered) increase, which should be contributed to the changes of the atomic packing type as discussed in Voronoi polyhedra. It should be emphasized that the packing around the smaller Co atoms has a narrower distribution of CNs (8-13), while the distribution around the larger $\mathrm{U}$ atoms is wide (10-17). The CNs' distribution of the 


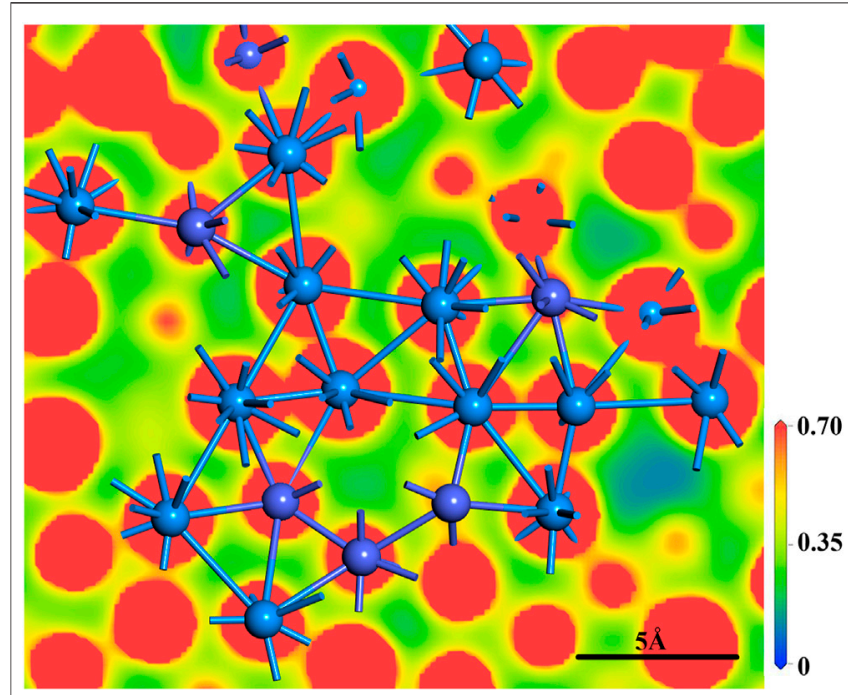

FIGURE 5 | The slice map of electron density of U-Co melts. Some U and $\mathrm{Co}$ atoms on the slice are shown in the figure. The cyan balls are $U$ atoms, and the purple balls are Co atoms. The unit of the scale plate is $|e| / \AA^{3}$.

$\mathrm{U}-\mathrm{Co}$ melts is similar to the r-MC results of the U-Fe metallic glasses (Zhang et al., 2012), which also show larger U-centered structures. The observation can be contributed to two perspectives based on the previous work (Cheng et al., 2013). One originates from the limited possibilities in the topological SROs of smaller atoms. A missing or extra neighbor will introduce an obvious deviation from the efficient packing scenario and thus higher energy costs (Miracle et al., 2003). The other is due to the stronger chemical effect between $U$ and Co atoms. The formation of U-Co bonds will be more energetic. The results suggest that Co-centered clusters are relatively more ordered and regular, which can be treated as building blocks in U-Co melts.

\section{Distribution of Electron Density}

As mentioned above results of structural indicators, two roles of $\mathrm{U}$ atoms, "chemical" and "topological", may be present in U-Co melts. To intuitively exhibit the roles of $U$ atoms, the electron density of U-Co melts is calculated. As shown in Figure 5, the electron density map also shows the SROs of U-Co melts consist of both longer $\mathrm{U}-\mathrm{U}$ bonds and shorter $\mathrm{U}-\mathrm{U}$ bonds, which is in agreement with the polyhedral analyses in Figure 3. The longer $\mathrm{U}-\mathrm{U}$ bonds are mainly metallic bonding characteristics. The shorter U-U bonds are connected by overlapping electron clouds, which are covalent bonding characteristics. It should be emphasized that although there are many dynamic configurations of U-Co melts, the configuration used to calculate the electron density is representative. Therefore, both the chemical and the topological effect of $U$ atoms are commonly present in U-Co melts. In some Ln-alloys/metals, a high-pressure condition will promote the delocalization of $4 f$-electron and the chemical effect of Ln-Ln, which induces the evolution of the atomic long or short-range order (Errandonea et al., 2000). However, for light Ans (Th $\sim \mathrm{Pu}$ ), the localization of $5 f$-electron is weaker than that of $4 f$-electron. The partial localization of 5f-electron was observed for the hightemperature behavior of $\gamma$-U (Yoo et al., 1998) and thermal expansion of $\delta$-Pu (Gouder et al., 2002). Namely, the partial localization of $U 5 f$-electron is responsible for the dual roles of $U$ atoms in U-Co melts, which are also the origin of the special SROs of U-Co melts.

\section{DISCUSSIONS}

In the present calculations, several structural indicators of U-Co melts are shown to discuss the special short-range orders. The first peaks of U-U PDFs consist of two subpeaks, which deviate from the predictions of the hard spheres model. The deviation suggests that $U$ atoms play dual roles, "chemical" and "topological" effects, to affect the SROs of U-Co melts. The dual roles are also confirmed by the Voronoi polyhedral analyses, which show two separate core-shell U-U distances. The behaviors of Co-centered clusters can be predicted by the solute-solvent model. Combined with the $\mathrm{CNs}$ results, we note that Co-centered clusters are the foundation of U-Co melts. By the electron density of U-Co melts, both metallic and covalent bonding characteristics are observed for U-U bonds. Finally, the dual roles of $\mathrm{U}$ atoms in $\mathrm{U}$-Co melts are contributed to the partial localization of $U 5 f$-electron. The result of the present work gives a theoretical view to analyze the origin of the SROs of Uranium alloys.

\section{DATA AVAILABILITY STATEMENT}

The original contributions presented in the study are included in the article/supplementary material, further inquiries can be directed to the corresponding authors.

\section{AUTHOR CONTRIBUTIONS}

All authors listed have made a substantial, direct, and intellectual contribution to the work and approved it for publication.

\section{FUNDING}

This research was funded by the National Natural Science Foundation of China (51871132), and Shandong Provincial Natural Foundation, China (ZR2019MB008). 


\section{REFERENCES}

Baenziger, N. C., Rundle, R. E., Snow, A. I., and Wilson, A. S. (1950). Compounds of Uranium with the Transition Metals of the First Long Period. Acta Cryst. 3, 34-40. doi:10.1107/S0365110X50000082

Blöchl, P. E. (1994). Projector Augmented-Wave Method. Phys. Rev. B 50, 17953-17979. doi:10.1103/PhysRevB.50.17953

Bowen, H. J. M., and Sutton, L. E. (1958). Tables of Interatomic Distances and Configuration in Molecules and Ions. London, UK: The Chemical Soc.

Cheng, Y. Q., Ding, J., and Ma, E. (2013). Local Topology vs. Atomic-Level Stresses as a Measure of Disorder: Correlating Structural Indicators for Metallic Glasses. Mater. Res. Lett. 1, 3-12. doi:10.1080/21663831.2012.722759

del Rio, B. G., González, L. E., and González, D. J. (2020). First Principles Study of Liquid Uranium at Temperatures up to 2050 K. J. Phys. Condens. Matter 32 (13), 304001. doi:10.1088/1361-648X/ab7f6f

Ding, J., Xu, M., Guan, P. F., Deng, S. W., Cheng, Y. Q., and Ma, E. (2014). Temperature Effects on Atomic Pair Distribution Functions of Melts. J. Chem. Phys. 140 (8), 064501. doi:10.1063/1.4864106

Drehman, A. J. (1985). "Density and Packing in Uranium Based Metallic Glasses," in Phase transitions in condensed systems-Experiments and Theory (Boston, USA: Cambridge University Press). doi:10.1557/proc-57-283

Drehman, A. J., and Poon, S. J. (1985). Anomalous Glass-Forming Ability of Uranium-Based Alloys. J. Non-Cryst. Sol. 76, 321-332. doi:10.1016/00223093(85)90008-0

Egami, T. (2011). Atomic Level Stresses. Prog. Mater. Sci. 56, 637-653. doi:10.1016/ j.pmatsci.2011.01.004

Egami, T., and Waseda, Y. (1984). Atomic Size Effect on the Formability of Metallic Glasses. J. Non-Cryst. Sol. 64, 113-134. doi:10.1016/00223093(84)90210-2

Elliott, R. O., and Giessen, B. C. (1982). On the Formation of Metallic Glasses Based on U, Np or Pu. Acta Metall. 30 (4), 785-789. doi:10.1016/0001-6160(82) 90076-1

Elliott, R. O., Koss, D. A., and Giessen, B. C. (1980). On the Characteristics of Amorphous U-Fe Alloys Formed by Liquid Quenching vs. Irradiation Techniques. Scr. Metall. 14, 1061-1065. doi:10.1016/0036-9748(80) 90206-9

Elliott, R. O., Smith, J. L., Finocchiaro, R. S., and Koss, D. A. (1981). Influence of Structure on the Properties of an Amorphous U-Fe alloy. Mater. Sci. Eng. 49, 65-69. doi:10.1016/0025-5416(81)90134-8

Errandonea, D., Boehler, R., and Ross, M. (2000). Melting of the Rare Earth Metals Andf-Electron Delocalization. Phys. Rev. Lett. 85, 3444-3447. doi:10.1103/ PhysRevLett.85.3444

Ganesh, P., and Widom, M. (2008). Ab Initiosimulations of Geometrical Frustration in Supercooled Liquid Fe and Fe-Based Metallic Glass. Phys. Rev. B 77 (10), 014205. doi:10.1103/PhysRevB.77.014205

Giessen, B. C., and Elliott, R. O. (1978). "Properties of Metallic Glasses Containing Actinide Metals. I. Thermal Properties of U-M Glasses ( $\mathrm{M}=\mathrm{V}$, $\mathrm{Cr}, \mathrm{Mn}, \mathrm{Fe}, \mathrm{Co}$, and Ni)," in Conference on Rapid Quenching, Brighton, UK, 3 July, 1978.

Gouder, T., Havela, L., Wastin, F., and Rebizant, J. (2002). $5 f$ Localization in Ultrathin $\mathrm{Pu}$ Layers. J. Nucl. Sci. Tech. 39, 49-55. doi:10.1080/ 00223131.2002.10875407

Hofman, G. L., Kim, Y. S., Finlay, M. R., Snelgrove, J. L., Hayes, S. L., Meyer, M. K., et al. (2003). "Recent Observations at the Postirradiation Examination of Low-Enriched U-Mo Miniplates Irradiated to High Burnup," in 25th International Meeting on Reduced Enrichment for Research and Test Reactors, Munich, Germany, 21-24 March 2004. (Chicago, USA: IAEA).

Huang, H. G., Zhang, P. G., Zhang, P., and Wang, Q. G. (2020). Comparison of Glass Forming Ability between U-Co and U-Fe Base Systems. Acta Metall. Sin. 56, 849-854. doi:10.11900/0412.1961.2019.00349

Huang, H. G., Ke, H. B., Wang, Y. M., Pu, Z., Zhang, P., Zhang, P. G., et al. (2016). Stable U-Based Metallic Glasses. J. Alloys Comp. 684, 75-83. doi:10.1016/ j.jallcom.2016.05.124

Huang, H. G., Wang, Y. M., Chen, L., Pu, Z., Zhang, P. G., and Liu, T. W. (2015). Study on Formation and Corrosion Resistance of Amorphous alloy in U-Co System. Acta Metall. Sin. 51, 623-630. doi:10.11900/ 0412.1961.2014.00580

Kaye, G. W. C., and Laby, T. H. (1993). Table of Physical and Chemical Constants. 15 th edition. London, UK: Longman.

Kresse, G., and Furthmüller, J. (1996). Efficiency of Ab-Initio Total Energy Calculations for Metals and Semiconductors Using a Plane-Wave Basis Set. Comput. Mater. Sci. 6, 15-50. doi:10.1016/0927-0256(96)00008-0

Kresse, G., and Joubert, D. (1999). From Ultrasoft Pseudopotentials to the Projector Augmented-Wave Method. Phys. Rev. B 59, 1758-1775. doi:10.1103/PhysRevB.59.1758

Li, F. F., Lu, L., Meng, X. D., Xiao, H., Ji, H. F., Zeng, R. G., et al. (2020). An Enhanced Hydrogen Corrosion by the $\mathrm{Ti}(\mathrm{C}, \mathrm{N})$ Inclusions in $\mathrm{U}-0.79$ wt\% $\mathrm{Ti}$ alloy. J. Alloys Comp. 820, 153124. doi:10.1016/j.jallcom.2019.153124

Manner, W. L., Lloyd, J. A., Hanrahan, R. J., and Paffett, M. T. (1999). An Examination of the Initial Oxidation of a Uranium-Base alloy (U-14.1 at.\% Nb) by $\mathrm{O}_{2}$ and $\mathrm{D}_{2} \mathrm{O}$ Using Surface-Sensitive Techniques. Appl. Surf. Sci. 150, 73-88. doi:10.1016/S0169-4332(99)00225-1

Massalski, T. B., Okamoto, H., Subramanian, P. R., and Kacprzak, L. (1990). Binary Alloy Phase Diagrams. Ohio, USA: ASM International.

Miracle, D. B. (2004). A Structural Model for Metallic Glasses. Nat. Mater. 3, 697-702. doi:10.1038/nmat1219

Miracle, D. B., Sanders, W. S., and Senkov, O. N. (2003). The Influence of Efficient Atomic Packing on the Constitution of Metallic Glasses. Phil. Mag. 83, 2409-2428. doi:10.1080/1478643031000098828

Miracle, D. B. (2006). The Efficient Cluster Packing Model - an Atomic Structural Model for Metallic Glasses. Acta Mater. 54, 4317-4336. doi:10.1016/ j.actamat.2006.06.002

Nosé, S. (1984). A Unified Formulation of the Constant Temperature Molecular Dynamics Methods. J. Chem. Phys. 81, 511-519. doi:10.1063/1.447334

Orlov, V. K. (2002). Fundamentals of the Physical Metallurgy of Uranium, Plutonium, and Their Alloys. Metal Sci. Heat Treat. 44, 11-12. doi:10.1023/ 1022596403845

Pan, S., Feng, S., Qiao, J., Dong, B., and Qin, J. (2015). The Atomic Structure of Liquid Fe-C Alloys. J. Alloys Comp. 648, 178-183. doi:10.1016/ j.jallcom.2015.06.252

Pan, S. P., Qin, J. Y., Gu, T. K., Yang, L., and Bian, X. F. (2009). Correlation between Local Structure of Melts and Glass Forming Ability for Fe78M9B13 (M=Nb, Si, and Zr) Alloys. J. Appl. Phys. 105 (4), 013531. doi:10.1063/1.3041473

Perdew, J. P., Burke, K., and Ernzerhof, M. (1996). Generalized Gradient Approximation Made Simple. Phys. Rev. Lett. 77, 3865-3868. doi:10.1103/ PhysRevLett.77.3865

Poon, S. J., Drehman, A. J., Wong, K. M., and Clegg, A. W. (1985). Superconductivity in Amorphous Actinide Alloys. Phys. Rev. B 31, 3100-3103. doi:10.1103/PhysRevB.31.3100

Porterfield, W. W. (1984). Inorginic Chemistry, a Unified Approach. Reading Masschusetts, USA: Addison Wesley Publishing Co.

Sheng, H. W., Liu, H. Z., Cheng, Y. Q., Wen, J., Lee, P. L., Luo, W. K., et al. (2007). Polyamorphism in a Metallic Glass. Nat. Mater 6, 192-197. doi:10.1038/ nmat 1839

Sheng, H. W., Luo, W. K., Alamgir, F. M., Bai, J. M., and Ma, E. (2006). Atomic Packing and Short-To-Medium-Range Order in Metallic Glasses. Nature 439, 419-425. doi:10.1038/nature04421

Shi, L.-L., Xu, J., and Ma, E. (2008). Alloy Compositions of Metallic Glasses and Eutectics from an Idealized Structural Model. Acta Material. 56, 3613-3621. doi:10.1016/j.actamat.2008.04.002

Shi, P., Li, F., Wu, Y., Ji, H., Li, R., and Wang, X. (2015). Effect of Alloyed Ti on the Microstructure and Corrosion Characteristics of a U-Ti alloy in a Hydrogen Environment. Corrosion Sci. 93, 58-62. doi:10.1016/ j.corsci.2015.01.005

Watson, R. E., and Bennett, L. H. (1991). Crystalline and Glassy Phases of Transition-Metal-Metalloid Systems. Phys. Rev. B 43, 11642-11652. doi:10.1103/PhysRevB.43.11642

Wiencek, T. C., and Prokofiev, I. G. (2000). "Low-enriched Uranium-Molybdenum Fuel Plate Development," in International Meeting on Reduced Enrichment for Research and Test Reactors, Las Vegas, NV, USA, 1-6 October 2000. (Las Vegas, NV, USA: RERTR). 
Xiang, S., Huang, H., and Hsiung, L. M. (2008). Quantum Mechanical Calculations of Uranium Phases and Niobium Defects in $\gamma$-uranium. J. Nucl. Mater. 375, 113-119. doi:10.1016/j.jnucmat.2007.11.003

Xu, H., Ke, H., Huang, H., Zhang, P., Pu, Z., Zhang, P., et al. (2018). U-based Metallic Glasses with superior Glass Forming Ability. J. Nucl. Mater. 499, 372-376. doi:10.1016/j.jnucmat.2017.11.043

Yoo, C.-S., Cynn, H., and Söderlind, P. (1998). Phase Diagram of Uranium at High Pressures and Temperatures. Phys. Rev. B 57, 10359-10362. doi:10.1103/ PhysRevB.57.10359

Zhang, Y., Mattern, N., Liang, T. X., Huang, Q., and Eckert, J. (2012). Atomic Packing and Short to Medium Range Order in a U-Fe Metallic Glass. Appl. Phys. Lett. 101, 021909. doi:10.1063/1.4736563

Zurek, A. K. (1999). Preshock-induced Phase Transition in Spalled U-0.75 wt.\% Ti. J. Nucl. Mater. 264, 155-160. doi:10.1016/S0022-3115(98)00462-0

Zurek, A. K. (1994). Spall Experiments and Microscopy of Depleted U-0.75\% Ti alloy. A Romanchenko Correction to a Spall Strength Calculation. J. Nucl. Mater. 211, 52-56. doi:10.1016/0022-3115(94)90280-1
Conflict of Interest: The authors declare that the research was conducted in the absence of any commercial or financial relationships that could be construed as a potential conflict of interest.

Publisher's Note: All claims expressed in this article are solely those of the authors and do not necessarily represent those of their affiliated organizations, or those of the publisher, the editors and the reviewers. Any product that may be evaluated in this article, or claim that may be made by its manufacturer, is not guaranteed or endorsed by the publisher.

Copyright (C) 2022 Zhang, Sun, Li and Wang. This is an open-access article distributed under the terms of the Creative Commons Attribution License (CC $B Y)$. The use, distribution or reproduction in other forums is permitted, provided the original author(s) and the copyright owner(s) are credited and that the original publication in this journal is cited, in accordance with accepted academic practice. No use, distribution or reproduction is permitted which does not comply with these terms. 